\title{
Simultaneous Determination of Florfenicol and Diclazuril in Compound Powder by RP-HPLC-UV Method
}

\author{
Leilei Guo, ${ }^{1}$ Xiangqin Tian, ${ }^{2}$ Shangran Shan, ${ }^{1}$ Jian Han, ${ }^{1}$ Xiaojun Shang, ${ }^{1}$ and Suying Ma ${ }^{1}$ \\ ${ }^{1}$ School of Pharmacy, Xinxiang Medical University, Jin Sui Road, Xinxiang 453003, China \\ ${ }^{2}$ Medicinal Experiment Center, Xinxiang Medical University, Jin Sui Road, Xinxiang 453003, China \\ Correspondence should be addressed to Suying Ma; suyingma@163.com
}

Received 2 April 2014; Revised 16 May 2014; Accepted 24 May 2014; Published 12 June 2014

Academic Editor: Pranav S. Shrivastav

Copyright (c) 2014 Leilei Guo et al. This is an open access article distributed under the Creative Commons Attribution License, which permits unrestricted use, distribution, and reproduction in any medium, provided the original work is properly cited.

\begin{abstract}
A RP-HPLC-UV method was developed and validated for simultaneous determination of florfenicol and diclazuril in compound powder. The separation involved using a SinoChoom ODS-BP $\mathrm{C}_{18}(5 \mu \mathrm{m}, 4.6 \mathrm{~mm} \times 250 \mathrm{~mm})$ analytical column. The mobile phase was a mixture of acetonitrile- $0.2 \%$ phosphoric acid ( $\mathrm{pH}$ was adjusted to 3.0 with triethylamine). The ratio of acetonitrile and $0.2 \%$ phosphoric acid in the mobile phase was $60: 40(\mathrm{v} / \mathrm{v})$ from 0 minutes to 6 minutes and $70: 30(\mathrm{v} / \mathrm{v})$ from 6.1 minutes to 15 minutes. The flow rate was $1 \mathrm{~mL} / \mathrm{min}$. The temperature of the analytical column was maintained at $30^{\circ} \mathrm{C}$. The detection was monitored at $225 \mathrm{~nm}$ and $277 \mathrm{~nm}$ for florfenicol and diclazuril, respectively. The excipients in the compound powder did not interfere with the drug peaks. The calibration curves of florfenicol and diclazuril were fairly linear over the concentration ranges between $50.0-$ $500.0 \mu \mathrm{g} / \mathrm{mL}(r=0.9995)$ and $10.0-100.0 \mu \mathrm{g} / \mathrm{mL}(r=0.9992)$, respectively. The RSD of both the intraday and interday variations was below $2.1 \%$ for florfenicol and diclazuril. The method was successfully validated according to International Conference on Harmonisation and proved to be suitable for the simultaneous determination of florfenicol and diclazuril in compound powder.
\end{abstract}

\section{Introduction}

Diclazuril chemically, 2-(4-Chlorophenyl)-2-[2, 6-dichloro4-(3, 5-dioxo-1, 2, 4-triazin-2-yl) phenyl] acetonitrile, is a broad-spectrum anticoccidial and antiprotozoal agent. It is widely used in chickens, turkeys, pigs, and cattle for prevention and treatment of coccidiosis $[1,2]$.

Florfenicol is a member of chloramphenicol and thiamphenicol family. The chemical name is 2,2 -dichloro- $\mathrm{N}$ $[(1 R, 2 S)$-3-fluoro-1-hydroxy-1-(4-methanesulfonylphenyl) propan-2-yl] acetamide. Florfenicol was widely used clinically now for the treatment of intestinal infections, respiratory tract infections, typhoid, and so on. Compared to thiamphenicol, florfenicol shows significant superiority in antibacterial spectrum, antibacterial activity, and considerably lower side effect; its antibacterial potency is 10 times higher than that of thiamphenicol [3-8].

However, due to the relatively poor water-soluble and low dissolution in gastric fluids of florfenicol and diclazuril, the two drugs show variation in bioavailability. Many researchers have made efforts to enhance the solubility of florfenicol and diclazuril by using organic solvents, solubilizer, or hydrotropy agent. Our previous studies have successfully prepared the solid dispersions of florfenicol and diclazuril with PEG6000 as carriers. It is possible to prepare the compound soluble powder with the two solid dispersions. The compound powder can play synergy roles for effective treatment of coccidiosis and prevention of the intestinal infections [9-11].

HPLC methods have been widely used to determine florfenicol and diclazuril in samples at present [12-17]. To our knowledge, no HPLC methods have been developed in the literature for determination of florfenicol and diclazuril in compound powder simultaneously. The literature was mainly focused on determination of the content of florfenicol and diclazuril one by one.

The main aim of this study is to develop and validate a sensitive, accurate, simple, and reproducible RPHPLC method according to International Conference on Harmonisation ( $\mathrm{ICH}$ ) to determine florfenicol and diclazuril simultaneously when combined in compound powder with the advantages of shorter retention time and run time [18]. 
<smiles>CS(=O)(=O)c1ccc([C@@H](O)[C@H](CF)NC(=O)C(Cl)Cl)cc1</smiles>

(a)<smiles>N#CC(c1ccc(Cl)cc1)c1c(Cl)cc(-n2ncc(=O)[nH]c2=O)cc1Cl</smiles>

(b)

FIGURE 1: Chemical structures of florfenicol (a) and diclazuril (b).

\section{Experimental}

2.1. Reagents and Chemicals. Florfenicol and diclazuril were received as gifts from Zhengzhou Zhongzhou pharmaceutical Co., Ltd (Zhengzhou, China), and the two standards were of over $99.5 \%$ purity (Figure 1). HPLC grade acetonitrile and other analytical grade chemicals were purchased from Xinshiji Chemicals Co., Ltd (Xinxiang, China). The deionized water in the study was purified with Smart2 Pure 12 UV/UF purification system (Thermo Fisher Scientific, USA).

2.2. Preparation of Florfenicol/Diclazuril Compound Powder. Florfenicol and diclazuril solid dispersions were prepared according to the method described by Dirikolu et al. and Norambuena et al. with PEG6000 as carriers [1315]. After drug content test, equivalent $5 \mathrm{~g}$ florfenicol and $0.5 \mathrm{~g}$ diclazuril were transferred to a mechanical blender, followed by mixing at least 10 minutes. Appropriate amount of soluble starch and glucose was added to the mixture and homogenized thoroughly for 20 minutes. The preparation was formulated to contain $5 \mathrm{~g}$ florfenicol and $0.5 \mathrm{~g}$ diclazuril in each $100 \mathrm{~g}$ compound powder and stored in the refrigerator at $4^{\circ} \mathrm{C}$ for further use.

\subsection{Preparation of Standard Stock Solutions and Working} Solutions. Stock standard solutions were prepared separately with acetonitrile to give a final concentration of $1.0 \mathrm{mg} / \mathrm{mL}$ for florfenicol and $100.0 \mu \mathrm{g} / \mathrm{mL}$ for diclazuril. The combined standard solutions were prepared with the above two solutions. Intermediate and working solutions were prepared by diluting the two stock solutions with the mobile phase. Calibration standard solutions were prepared in the concentration range from 50.0 to $500.0 \mu \mathrm{g} / \mathrm{mL}$ for florfenicol and from 10.0 to $100.0 \mu \mathrm{g} / \mathrm{mL}$ for diclazuril and injected into the system in triplicate. The chromatogram peak area of each drug concentration was calculated. The regression of the drug concentration versus the peak area was obtained.

2.4. Liquid Chromatographic Conditions. The liquid chromatographic analyses were performed using a Shimadzu system that was comprised of LC-20AT pumps and SPD 20A UV-visible absorbance detector connected to Shimadzu Spin Chrome software. Chromatographic separation was achieved on a reversed-phase ODS-BP $\mathrm{C}_{18}$ column $(5 \mu \mathrm{m}$,
$4.6 \mathrm{~mm} \times 250 \mathrm{~mm}$ ); an injection volume of $20 \mu \mathrm{L}$ was optimized in the method via a Rheodyne syringe.

The mobile phase under gradient mode was a mixture of acetonitrile $-0.2 \%$ phosphoric acid ( $\mathrm{pH}$ was adjusted to 3.0 with triethylamine). The ratio of acetonitrile and $0.2 \%$ phosphoric acid in the mobile phase was $60: 40(\mathrm{v} / \mathrm{v})$ from 0 minutes to 6 minutes and $70: 30(\mathrm{v} / \mathrm{v})$ from 6.1 minutes to 15 minutes. The mobile phase was degassed by an ultrasonic bath and filtered through a $0.45 \mu \mathrm{m}$ membrane filter under vacuum. The eluents were detected at $225 \mathrm{~nm}$ from 0 minutes to 6.0 minutes and $277 \mathrm{~nm}$ from 6.1 minutes to 15.0 minutes. The flow rate was $1.0 \mathrm{~mL} / \mathrm{min}$. All determinations were performed at $30^{\circ} \mathrm{C}$.

2.5. Quantification of Florfenicol and Diclazuril in Compound Powder. $1.0 \mathrm{~g}$ compound powder was accurately weighted and transferred into a $50 \mathrm{~mL}$ volumetric flask. The powder was dissolved and made up to volume with mobile phase. $5 \mathrm{~mL}$ solution was withdrawn and transferred into another $25 \mathrm{~mL}$ volumetric flask. The concentrations of the solution were $200.0 \mu \mathrm{g} / \mathrm{mL}$ and $20.0 \mu \mathrm{g} / \mathrm{mL}$ for florfenicol and diclazuril, respectively. A $20 \mu \mathrm{L}$ aliquot of the sample solution was injected into the chromatographic system three times under optimized chromatographic conditions. The peak area was measured at $225 \mathrm{~nm}$ from 0 minutes to 6.0 minutes and at $277 \mathrm{~nm}$ from 6.1 minutes to 15.0 minutes for florfenicol and diclazuril, respectively. Drug concentrations of the samples were determined by interpolation from calibration plots of each drug previously obtained.

2.6. Method Validation. The proposed method was validated in terms of parameters of specificity, linearity, sensitivity, accuracy, precision, and reproducibility according to $\mathrm{ICH}$.

The specificity of the method was demonstrated by comparing chromatograms of compound powder sample (florfenicol $200.0 \mu \mathrm{g} / \mathrm{mL}$ and diclazuril $20.0 \mu \mathrm{g} / \mathrm{mL}$ ) and blank excipients sample without florfenicol and diclazuril. All the samples were analyzed and recorded to ensure the absence of interfering peaks.

The linearity of the method was evaluated with florfenicol and diclazuril working solutions at eight different concentrations. The concentration was $50.0-500.0 \mu \mathrm{g} / \mathrm{mL}$ for florfenicol and $10.0-100.0 \mu \mathrm{g} / \mathrm{mL}$ for diclazuril, respectively. 


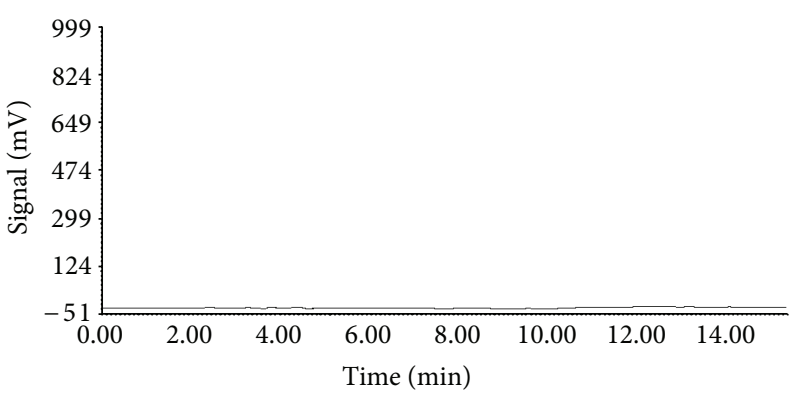

(a)

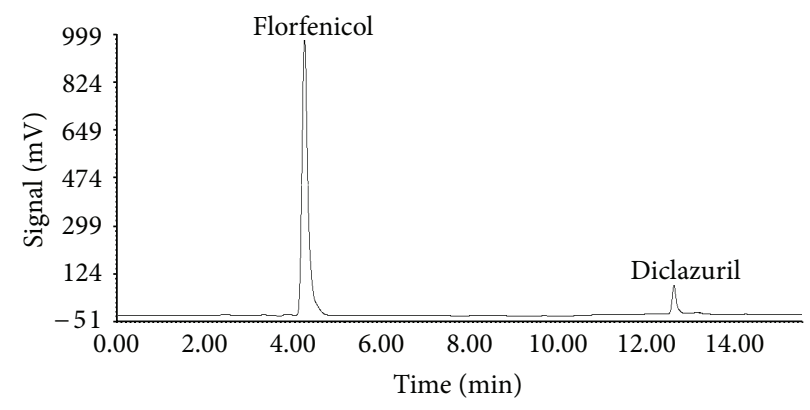

(b)

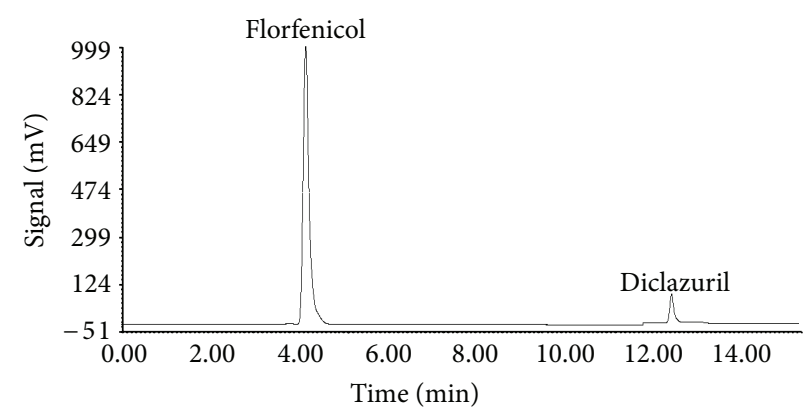

(c)

FIGURE 2: Typical HPLC chromatograms of (a) blank excipients sample, (b) compound powder sample with florfenicol and diclazuril (c) standard solutions sample with florfenicol $(200.0 \mu \mathrm{g} / \mathrm{mL})$ and diclazuril $(20.0 \mu \mathrm{g} / \mathrm{mL})$.

All the samples prepared for linearity were injected into chromatographic system $(n=3)$. The responses were measured as peak area.

The sensitivity of the method was tested with limit of detection (LOD) and limit of quantification (LOQ). The LOD and LOQ were expressed as the analytes concentration which generates a signal corresponding to three and ten standard deviations, respectively, above the mean blank signal.

The accuracy of the method was assessed by comparing the percent analytes recovered by the proposed method at three concentration levels (florfenicol 160.0, 200.0, and $240.0 \mu \mathrm{g} / \mathrm{mL}$ and diclazuril 16.0, 20.0, and $24.0 \mu \mathrm{g} / \mathrm{mL}$ ).

The precision of the method was checked by repeatability of injection, repeatability (intraday), intermediate precision (interday), and reproducibility. Injection repeatability was studied by calculating percent relative standard deviation (\% RSD) for ten determinations of peak area of florfenicol $(200.0 \mu \mathrm{g} / \mathrm{mL})$ and diclazuril $(20.0 \mu \mathrm{g} / \mathrm{mL})$ performed on the same day. The same solutions were injected in triplicate for both intraday and interday variations.

\section{Results}

3.1. Method Validation. The specificity was evaluated by analyzing the chromatograms of blank excipients sample and compound powder sample (florfenicol $200.0 \mu \mathrm{g} / \mathrm{mL}$ and diclazuril $20.0 \mu \mathrm{g} / \mathrm{mL}$ ). From the UV-visible spectra, florfenicol had maximum absorption at $225 \mathrm{~nm}$ and diclazuril had maximum absorption at $277 \mathrm{~nm}$. Thus, $225 \mathrm{~nm}$ and $277 \mathrm{~nm}$ were selected as detection wavelengths. The typical HPLC chromatograms under optimum conditions were shown in Figure 2. The retention times of florfenicol and diclazuril at a flow rate of $1.0 \mathrm{~mL} / \mathrm{min}$ were $4.10 \mathrm{~min}$ and $12.20 \mathrm{~min}$, respectively. Analyte peaks were well resolved and free from tailing ( $<1.5$ for both analytes). The excipients in the compound powder did not interfere with the detection of florfenicol and diclazuril.

The calibration curves obtained by plotting peak area against concentration were linear over the concentration range of $50.0-500.0 \mu \mathrm{g} / \mathrm{mL}$ for florfenicol and 10.0 $100.0 \mu \mathrm{g} / \mathrm{mL}$ for diclazuril at eight different concentrations, respectively. The correlation coefficient values were over 0.9990. Typical regression equations were calculated as follows: $y=44.831 x+163.73$ for florfenicol and $y=37.01 x+$ 25.807 for diclazuril, where $y$ is peak area based on three parallel measurements and $x$ is the concentration $(\mu \mathrm{g} / \mathrm{mL})$ of florfenicol or diclazuril standard solution. The correlation coefficients indicate a good linear relationship between peak area and concentration over a wide range.

Under the developed HPLC conditions, the LOD for florfenicol or diclazuril was 2.5 and $1.5 \mu \mathrm{g} / \mathrm{mL}$, respectively, which is the concentration that yields an S/N of 3, while LOQ was 5.0 and $2.5 \mu \mathrm{g} / \mathrm{mL}$, respectively [19].

Mean recovery for florfenicol or diclazuril was $99.76 \pm$ $0.38 \%$ and $100.24 \pm 0.56 \%$, respectively. The intra- and interday RSD values were lower than $2.1 \%$. The low values of RSD revealed satisfactory precision and accuracy of this present method. Reproducibility was checked by having samples analyzed by another analyst using the same instrument and the same laboratory. There was no significant difference 
between the RSD values indicating the proposed method was reproducible.

\subsection{Content of Florfenicol or Diclazuril in Compound Powder.}

The proposed method was applied to simultaneously determine the florfenicol and diclazuril in compound powder. The results of the assay yielded $98.49 \pm 0.33 \%$ for florfenicol and $101.46 \pm 0.58 \%$ for diclazuril indicating that the method was selective and accurate for the simultaneous determination of florfenicol and diclazuril without interference from the excipients in the compound powder dosage form.

\section{Discussion}

This study was essentially focused on the simultaneous determination of coformulated florfenicol or diclazuril in compound powder. The solid dispersions of florfenicol and diclazuril have first been prepared with PEG6000 as carriers, and then the soluble powder was made with the two solid dispersions, soluble starch, and glucose. The soluble starch and glucose are not only used as excipients in pharmaceutical preparations, but they also provide the animals with nutrition and energy. Further study will be published in another research paper later.

Florfenicol and diclazuril solid dispersions were soluble in organic solvents including methanol and acetonitrile [20, 21]. The use of mobile phase as reagent provided minimal impurities and better separation.

Chromatographic conditions were studied in order to achieve the best separation and retention for the analytes, minimizing analysis time.

Several mobile phase systems including methanol, acetonitrile, and phosphoric acid solutions of different proportions have been tested in this study. A mixture of acetonitrile- $0.2 \%$ phosphoric acid ( $\mathrm{pH}$ was adjusted to 3.0 with triethylamine) with the ratio ranged from $60: 40(\mathrm{v} / \mathrm{v})$ to $70: 30(\mathrm{v} / \mathrm{v})$ was selected as the optimum mobile phase for baseline separation, symmetrical peak, and shorter retention time. Triethylamine was used to adjust the $\mathrm{pH}$ for better separation and decrease the peak tailing of compounds in the proposed method. Diclazuril could not be detected with the UV detector if the ration of acetonitrile in the mobile phase was too low.

The UV detector was set at $225 \mathrm{~nm}$ for florfenicol and at $277 \mathrm{~nm}$ for diclazuril. Under these conditions, elution of analytes was completed in less than $13.0 \mathrm{~min}$. Retention times of florfenicol and diclazuril were $4.10 \mathrm{~min}$ and $12.20 \mathrm{~min}$, respectively. The chromatograms were evaluated on the basis of peak areas of the two analytes.

The method was validated according to ICH guidelines with the parameters of specificity, linearity, sensitivity, accuracy, precision, and reproducibility. The resolved analytes peaks without tailing between florfenicol and diclazuril showed the efficiency of the method to identify and determine each analyte at the same time with no interference. The accuracy, sensitivity, precision, and reproducibility data show that the method is accurate within the desired ranges.
The RP-HPLC method coupled with UV detector has been successfully developed. The method is very suitable for the application in a large scale because the involved instrument is very cheap and popular in any analysis laboratory.

\section{Conclusion}

In summary, a sensitive, simple, and selective RP-HPLC method has been developed and validated for the simultaneous determination of florfenicol and diclazuril in compound powder following the ICH guidelines. This is the first report on the simultaneous quantitation of this drug combination with high clinical relevance. The method will be helpful for simultaneous determination of florfenicol and diclazuril in compound powder and can be reliably used by almost every drug analysis laboratory.

\section{Conflict of Interests}

The authors declare that there is no conflict of interests regarding the publication of this paper.

\section{Acknowledgments}

The authors would like to acknowledge the financial support provided by the Education Department Henan Province (Foundation no: 13A350851), Xinxiang medical university, and Ministry of Education of the People's Republic of China (Foundation no: 201310472023).

\section{References}

[1] L. R. McDougald and B. P. Seibert, "Residual activity of anticoccidial drugs in chickens after withdrawal of medicated feeds," Veterinary Parasitology, vol. 74, no. 2-4, pp. 91-99, 1998.

[2] M. A. Taylor, J. Catchpole, J. Marshall, R. N. Marshall, and D. Hoeben, "Histopathological observations on the activity of diclazuril (Vecoxan) against the endogenous stages of Eimeria crandallis in sheep," Veterinary Parasitology, vol. 116, no. 4, pp. 305-314, 2003.

[3] B. R. Hoar, M. D. Jelinski, C. S. Ribble, E. D. Janzen, and J. C. Johnson, "A comparison of the clinical field efficacy and safety of florfenicol and tilmicosin for the treatment of undifferentiated bovine respiratory disease of cattle in western Canada," The Canadian Veterinary Journal, vol. 39, no. 3, pp. 161-166, 1998.

[4] S. Sadeghi and M. Jahani, "Selective solid-phase extraction using molecular imprinted polymer sorbent for the analysis of Florfenicol in food samples," Food Chemistry, vol. 141, no. 2, pp. 1242-1251, 2013.

[5] R. L. Pentecost, A. J. Niehaus, N. A. Werle, and J. Lakritz, "Pharmacokinetics of florfenicol after intravenous and intramuscular dosing in llamas," Research in Veterinary Science, vol. 95, no. 2, pp. 594-599, 2013.

[6] K. J. Varma, P. E. Adams, T. E. Powers, J. D. Powers, and J. F. Lamendola, "Pharmacokinetics of florfenicol in veal calves," Journal of Veterinary Pharmacology and Therapeutics, vol. 9, no. 4, pp. 412-425, 1986.

[7] C. W. Booker, G. K. Jim, P. T. Guichon, O. C. Schunicht, B. E. Thorlakson, and P. W. Lockwood, "Evaluation of florfenicol 
for the treatment of undifferentiated fever in feedlot calves in western Canada," The Canadian Veterinary Journal, vol. 38, no. 9, pp. 555-560, 1997.

[8] C. Graziani, L. Busani, A. M. Dionisi et al., "Antimicrobial resistance in Salmonella enterica serovar Typhimurium from human and animal sources in Italy," Veterinary Microbiology, vol. 128, no. 3-4, pp. 414-418, 2008.

[9] S. Ma and X. Shang, "Preparation and characterization of florfenicol-polyethyleneglycol 4000 solid dispersions with improved solubility," Asian Journal of Chemistry, vol. 24, no. 7, pp. 3059-3063, 2012.

[10] S. Ma and X. Shang, "Study on diclazuril polyethylene glycol 6000 solid dispersions with improved solubility," Asian Journal of Chemistry, vol. 24, no. 11, pp. 5202-5204, 2012.

[11] S. Y. Ma, R. Y. Dong, X. J. Shang, and F. L. Yan, "Preparation and characterization of florfenicol-polyethyleneglycol 6000 solid dispersions," Journal of Hunan Agricultural University (Natural Sciences), vol. 36, no. 5, pp. 589-593, 2010.

[12] Z. Shi, Q. Ge, J. Lu et al., "Comparison of pretreatment methods for the simultaneous determination of diclazuril and toltrazuril residues in chicken tissues," Chinese Journal of Chromatography, vol. 27, no. 3, pp. 303-307, 2009.

[13] L. Dirikolu, F. Lehner, C. Nattrass et al., "Diclazuril in the horse: its identification and detection and preliminary pharmacokinetics," Journal of Veterinary Pharmacology and Therapeutics, vol. 22, no. 6, pp. 374-379, 1999.

[14] L. Norambuena, N. Gras, and S. Contreras, "Development and validation of a method for the simultaneous extraction and separate measurement of oxytetracycline, florfenicol, oxolinic acid and flumequine from marine sediments," Marine Pollution Bulletin, vol. 73, no. 1, pp. 154-160, 2013.

[15] K. Xie, L. Jia, Y. Yao et al., "Simultaneous determination of thiamphenicol, florfenicol and florfenicol amine in eggs by reversed-phase high-performance liquid chromatography with fluorescence detection," Journal of Chromatography B: Analytical Technologies in the Biomedical and Life Sciences, vol. 879, no. 23, pp. 2351-2354, 2011.

[16] A. Cañas, S. Valdebenito, and P. Richter, "A new rotating-disk sorptive extraction mode, with a copolymer of divinylbenzene and $\mathrm{N}$-vinylpyrrolidone trapped in the cavity of the disk, used for determination of florfenicol residues in porcine plasma," Analytical and Bioanalytical Chemistry, vol. 406, no. 8, pp. 2205-2210, 2014.

[17] J. Hayes, "Determination of florfenicol in fish feeds at high inclusion rates by HPLC-UV," Journal of AOAC International, vol. 96, no. 1, pp. 7-11, 2013.

[18] “Text on validation of analytical procedures Q2A," in Proceeding of the International Conference on Harmonisation of Technical Requirements for Registration of Pharmaceuticals for Human Use, London, UK, 1994.

[19] E. N. Evaggelopoulou and V. F. Samanidou, "Development and validation of an HPLC method for the determination of six penicillin and three amphenicol antibiotics in gilthead seabream (Sparus Aurata) tissue according to the European Union Decision 2002/657/EC," Food Chemistry, vol. 136, no. 34, pp. 1322-1329, 2013.

[20] Florfenicol [homepage on the Internet], http://hanyu.iciba. com/wiki/5592977.shtml.

[21] Diclazuril [homepage on the Internet], http://baike.baidu.com/ view/1076193.htm?fr=aladdin. 

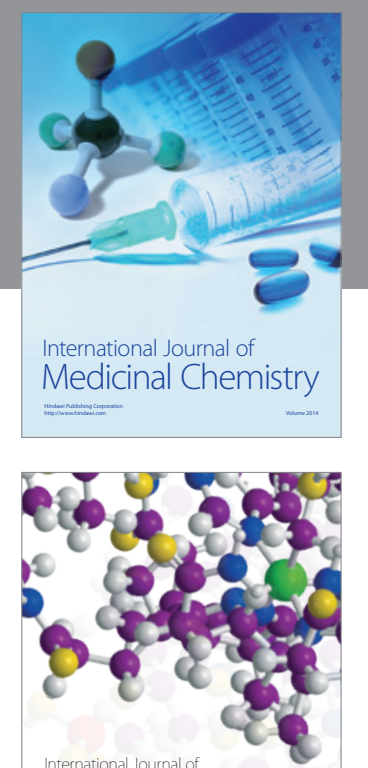

\section{Carbohydrate} Chemistry

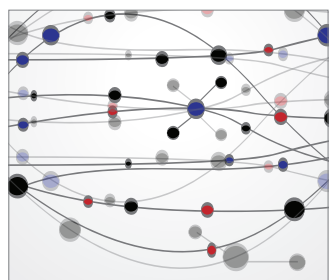

The Scientific World Journal
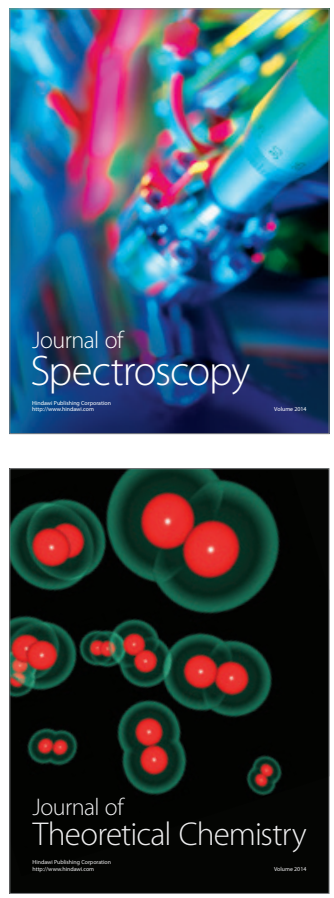
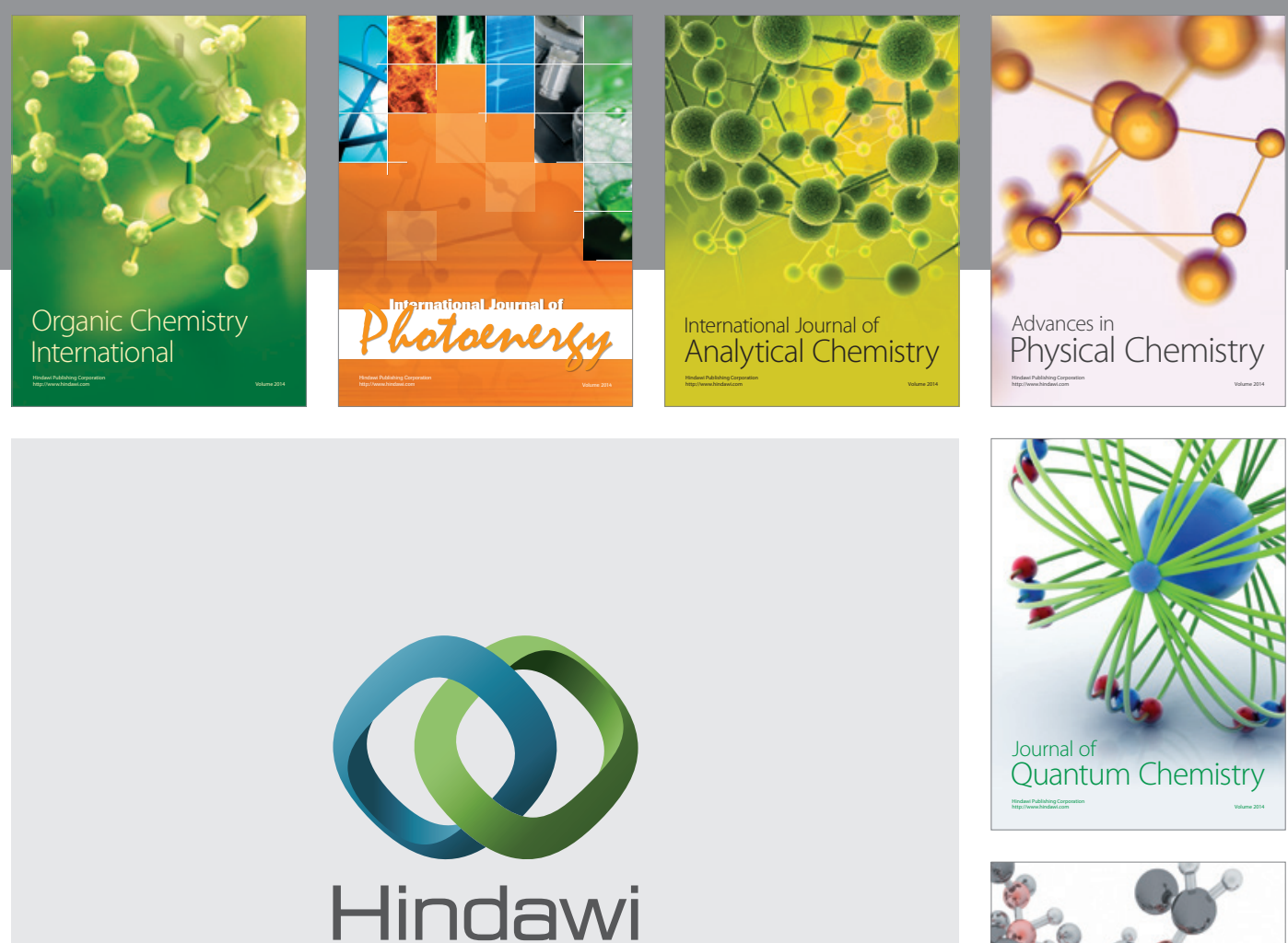

Submit your manuscripts at

http://www.hindawi.com

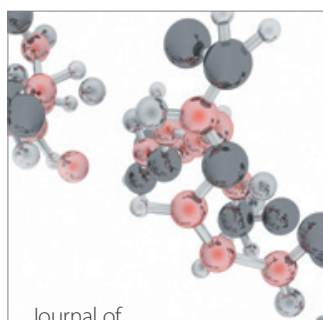

Analytical Methods

in Chemistry

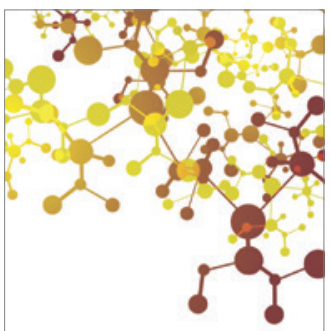

Journal of

Applied Chemistry

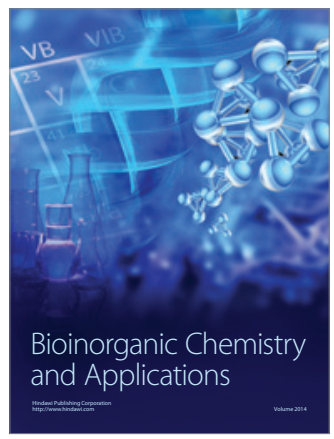

Inorganic Chemistry
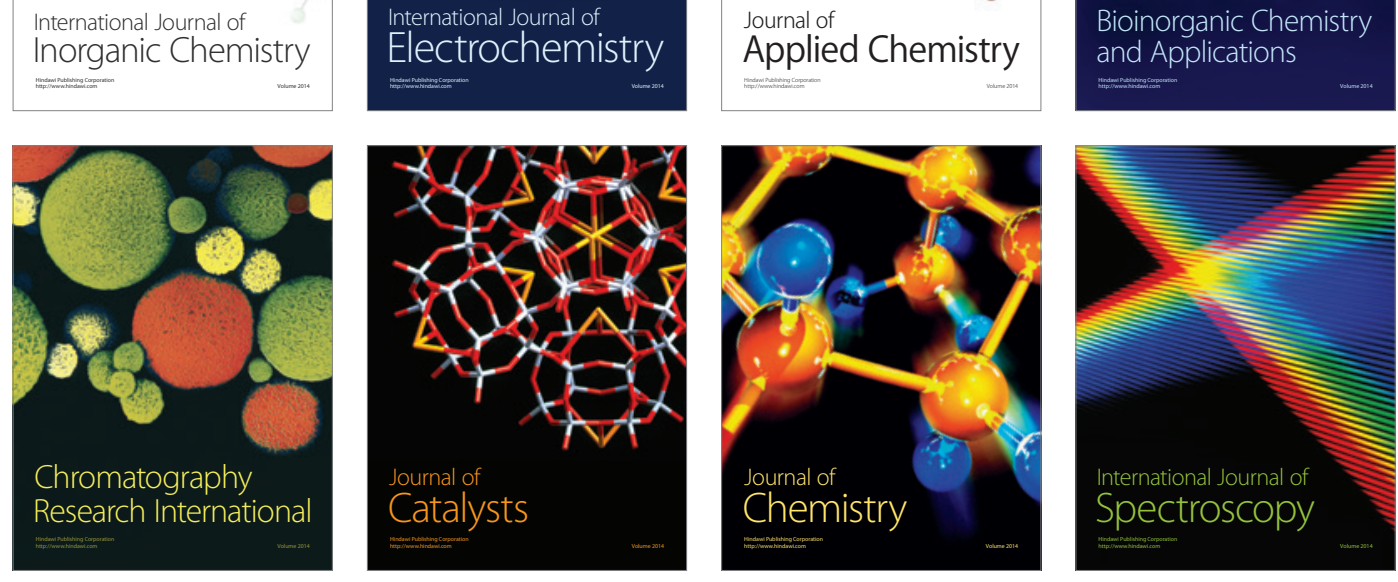